\title{
New biomimetic approach to the aircraft wing structural design based on aeroelastic analysis
}

\author{
D. GAWEL*, M. NOWAK, H. HAUSA, and R. ROSZAK \\ ${ }^{1}$ Chair of Virtual Engineering, Poznan University of Technology, 24 Jana Pawla II, 60-965 Poznan, Poland
}

\begin{abstract}
This paper presents a new biomimetic approach to the structural design. For the purpose of aircraft wing design the numerical environment combining simultaneous structural size, shape, and topology optimization based on aeroelastic analysis was developed. For the design of aircraft elements the optimization process must be treated as a multi-load case task, because during the fluid structure interaction analysis each step represents a different structural load case. Also, considering different angles of attack, during the CFD computation each result is considered. The method-specific features (such as domain independence, functional configurations during the process of optimization, and multiple load case solution implemented in the optimization scenario) enable the optimal structural form. To illustrate the algorithm functionality, the problem of determining the optimal internal wing structure was presented. The optimal internal wing structure resulting from aeroelastic computation with different angles of attack has been presented.
\end{abstract}

Key words: optimization, fluid-structure interaction, coupling techniques, biomimetics.

\section{Introduction}

Recently the role of optimization in aerospace and automotive industry has increased becoming a significant part of the design process. Topology optimization (TO) $[1,2]$ is perceived to be a next step in computer aided engineering (CAE) after the finite element method (FEM). It ensures a great mass reduction, leading to material savings, while simultaneously keeping the assumed strength properties of the final product. Topology optimization is still a slightly new, but rapidly developing, field of knowledge that needs further verification. Despite that, many companies have decided to implement this innovative technique into their design process, expecting future profits.

This paper presents an alternative approach to topology optimization based on trabecular bone remodeling. The new biomimetic optimization method applied to the fluid-structure interaction problem contributes new possibilities for optimization due to unique features including simultaneous size, shape, and topology optimization. The algorithm developed will be presented on the NACA0012 wing example. In addition to the optimization method, the algorithm of combining multiple load cases (different pressure distributions) on the surface will be discussed.

\section{State of the art}

In 1996 Balabanov and Haftka used discrete ground structures in truss topology optimization for designing the internal struc-

\footnotetext{
*e-mail: dominik.r.gawel@doctorate.put.poznan.pl
}

Manuscript submitted 2016-10-26, revised 2017-02-21 and 2017-03-15, initially accepted for publication 2017-03-22, published in October 2017. ture of a wing. Their solution computed the pressure distribution for an analyzed shape, but missed coupling.

Another approach to wing optimization refers to the reduction of skin thickness, which was done by Wang and Williams [3]. The wing was divided into dozens of panels, modeled as membranes. The wing's weight was reduced along with the increased stiffness of the whole model, stemming from the modification of the membranes thickness.

In the papers of Krog [4, 5] the optimization of Airbus A380 ribs was treated as a two-step process. Potential geometry was reduced using standard topology optimization, and then the geometrical model was created for structural optimization purposes. Finally, the optimal structure was transformed into a CAD model which was afterwards verified.

Stettner and Schuhmacher [6] also referred to topological and structural optimizations for designing the rear part of a military transport aircraft fuselage. The whole assembly as well as its frame has been analyzed with the same optimization methods within a two-stage process. The results were obtained separately for bending load, torsional load, and pressure exerted on side walls. Additionally, authors attempted to compile the individual results into a single form. However, it has to be emphasized that presented solution did not result from multi-load case calculations.

Maute and Allen [7] suggested implementation of structural optimization methods in combination with an aeroelastic simulation. The problem was presented on the example of a slim wing model used for the two-dimensional topological optimization. The obtained configuration presented a conceptual distribution of the material inside the wing subjected to the impact of surrounding flow. The internal structure significantly differed from one we might expect on the basis of an analysis of the regular pressure distribution over a wing surface. 
Liu et al. [8] examined wing cross-section on its fixed part with the use of topological optimization. An assumed force distribution over a three-dimensional wing has been limited to a single rib - the optimization domain.

James and Martins [9] analyzed the internal wing structure using the level set method. An additional element was incorporated: consideration of local stresses in order to introduce strength limitations into the area. An artificial load was applied to a three-dimensional area, with an assumed initial configuration. Thus, the resulting material distribution depends on the initial structure.

Oktay et al. [10] combined results from a computational fluid dynamics (CFD) simulation on the computational solid mechanics (CSM) grid for the structure, which was used to conduct a topological optimization. The data from the flow grid was interpolated onto the grid for the structure by means of bi-line interpolation. The optimization process itself was conducted on one of the cross sections selected by the authors. Oktay et al. [11] also used the solid isotropic material with penalization (SIMP) method for optimization of the entire wing box space, looking for new possible configurations.

This article presents a new biomimetic optimization method applied to the fluid-structure interaction problem. Presented algorithm contributes new possibilities for optimization due to unique features including simultaneous size, shape, and topology optimization. The volume resulting from the presented optimization procedure is not assumed before optimization, but is related to the assumed strain energy density and material strength properties, as opposed to the method presented by Sleesongsom and Bureerat [12]. Moreover, the method presented herein merges multiple load configurations without overlapping results.

\section{Biomimetic optimization method}

Regarding the Wolff Law, established in 19th century after many experiments, conclusions were drawn confirming bone's capacity to adapt itself to mechanical stimulation. Nowadays scientists are certain that the trabecular bone tends to mechanical optimum by managing the number and organization of its internal structures - beams. In literature we can identify multiple bone remodeling models [13-16], which simplify the problem by treating the bone as a complex continuum material with specific properties determined by its load history.

On the other hand, due to increasing computing power and parallel computation techniques, it is possible to imitate the bone adaptation process using a linear model of the trabecula, resembling a real topology of the trabecular bone $[14,16,17]$. To achieve proper and effective simulation results, two crucial laws of trabecular bone adaptation phenomenon need to be preserved: first, to obtain rebuilding balance (homeostasis) a mechanical stimulation is needed (mechanosensitivity), and second, the formation and resorption processes need to occur on the structure surface only (surface adaptation) [18].

A developed topology optimization system based on the bone remodeling simulation and finite element method (FEM) calculations connects three general steps: FEM preprocessing, FEM solution, and optimization procedures. Regarding the second law of the trabecular bone adaptation phenomenon, the remodeling algorithm is based on the strain energy density (SED) distribution, to achieve the proper mechanical loading stimulation. This evolving model reflects real trabecular bone structures: beams (assumed to be an isotropic linear elastic material) and marrow spaces (treated as voids). This approach imitates the real bone remodeling process in a complex way by controlling not only the volumetric mesh, but also the surface of the trabecular network.

3.1. Trabecular bone remodelling process. The trabecular bone remodeling process is an excellent example of the structural optimization problem. There are many models of bone remodeling. Most of them represent the continuation of the Roux's idea of biological regulatory process [19-23]. In the "regulatory model" developed by Huiskes [13, 24, 25], the concept of tissue adaptation is based on the assumption of the existence of homeostasis (perfect balance between bone gain and loss).

This equilibrium can occur only in the presence of mechanical stimulation. The network of osteocytes plays the role of sensors detecting mechanical energy distribution along trabecu lar bone tissue. The model postulates strain energy density (SED) on the surface of trabecular bone as a scalar measure of mechanical stimulation and a distinguished value of SED, corresponding to bone remodeling homeostasis. Thus, the regulatory mechanism is responsible for the remodeling process in the trabecular bone on a single cell level. The model assumes also that small deviations from this distinguished energy value do not substantially influence the remodeling phenomenon (Carter's "lazy zone" [21]). Only significant changes in the mechanical stimulation result in bone loss or gain. The "regulatory model" is described according to [24] by the following equations:

$$
\begin{gathered}
\frac{d E}{d t}=C_{e}\left(U-\left(U_{h}+s\right)\right) \quad \text { for } \quad U>\left(U_{h}+s\right), \\
\frac{d E}{d t}=0 \quad \text { for } \quad\left(U_{h}-s\right) \leq U \leq\left(U_{h}+s\right), \\
\frac{d E}{d t}=C_{e}\left(U+\left(U_{h}+s\right)\right) \text { for } U<\left(U_{h}-s\right),
\end{gathered}
$$

where: $E$ denotes Young's modulus of the tissue, $U_{h}$ is the strain energy density (SED) value corresponding to homeostasis of bone loss and gain, $2 s$ is the size of the "lazy zone", $C_{e}$ is a constant value on structural surface.

3.2. The principle of constant strain energy density. The essential assumption of the "regulatory model" is the existence of homeostasis in the remodeling process, described by the distinguished value of SED. But SED (as an energy measure) is also of a prime importance in optimization research, far from biomechanical applications [26-28]. Pedersen [28] presented 
the considerations of the optimization of structural shape by minimizing the strain energy. Defining the total potential as a sum of elastic energy and work of external forces [28]:

$$
\Pi=U_{\varepsilon}+U_{e x t}
$$

the derivative of the total potential $\Pi$ with respect to an arbitrary parameter $h$ is:

$$
\frac{d \Pi}{d h}=\frac{\partial \Pi}{\partial h}+\frac{\partial \Pi}{\partial \varepsilon} \frac{d \varepsilon}{d h},
$$

and with respect to the virtual work principle:

$$
\frac{\partial \Pi}{\partial \varepsilon}=0
$$

for design independent external loads:

$$
\frac{\partial U_{e x t}}{\partial \varepsilon}=0
$$

the derivative of the total potential:

$$
\frac{d \Pi}{d h}=\frac{\partial U_{\varepsilon}}{\partial h} .
$$

For a local design parameter $h_{e}$ that only changes the design in the domain $e$ of the structure:

$$
\frac{\partial U_{\varepsilon}}{\partial h_{e}}=\frac{\partial\left(\bar{u}_{e} V_{e}\right)}{\partial h_{e}},
$$

where: $\bar{u}_{e}$ is the mean strain energy density in the domain of $e$, $V_{e}$ is the corresponding volume.

Assuming two parameters $h_{i}, h_{j}$ and a constant total volume $V$ of the structure:

$$
\Delta V=\frac{d V}{d h_{i}} \Delta h_{i}+\frac{d V}{d h_{j}} \Delta h_{j}=0,
$$

we arrive at the increment of the elastic energy:

$$
\Delta U_{\varepsilon}=\frac{d U_{\varepsilon}}{d h_{i}} \Delta h_{i}+\frac{d U_{\varepsilon}}{d h_{j}} \Delta h_{j}
$$

for design independent loads, and it follows from (9) that only the local energies are involved:

$$
\begin{array}{r}
\Delta U_{\varepsilon}=\bar{u}_{i} \frac{d V_{i}}{d h_{i}} \Delta h_{i}+\bar{u}_{j} \frac{d V_{j}}{d h_{j}} \Delta h_{j}, \\
\Delta U_{\varepsilon}=-\left(\bar{u}_{i}-\bar{u}_{j}\right) \frac{d V_{i}}{d h_{i}} \Delta h_{i} .
\end{array}
$$

With the assumption of a constant volume the necessary condition for optimality:

$$
\Delta U_{\varepsilon}=0
$$

leads to the conclusion that the strain energy densities must be equal to:

$$
\bar{u}_{i}=\bar{u}_{j}
$$

Similarly, with all design parameters:

$$
\Delta V=\sum_{e} \frac{d V_{e}}{d h_{e}} \Delta h_{e}
$$

the total energy change equation:

$$
\Delta U_{e}=\sum_{e} u_{e} \frac{d V_{e}}{d h_{e}} \Delta h_{e}
$$

leads to the conclusion that a necessary condition for the optimality $\Delta U=0$ with constraint $\Delta V=0$ is a constant value of the strain energy density. Thus, for the stiffest design [26, 28] the energy density along the shape to be designed must be constant:

$$
u_{\varepsilon s}=\text { const. }
$$

Equation (17) proves that SED used as a remodeling signal in the Huiskes's remodeling model is a very good choice. The natural, metabolic trabecular bone remodeling process is, in this context, a practical realization of the structural optimization.

By analogy to the bone remodeling process described above, the following assumptions have been made (the variables have the same meaning as in equations (1-3)):

Add some amount of material onto the surface of the structure for

$$
U>\left(U_{h}+s\right)
$$

No action - "lazy zone" for

$$
\left(U_{h}-s\right)<U<\left(U_{h}+s\right) .
$$

Remove some amount of material from the surface of the structure for

$$
U<\left(U_{h}-s\right)
$$

In this way, the process of structural optimization mimics the real biological process of mechanical adaptation of trabecular bone. The material in virtual space is (just like the tissue) added onto or removed from the surface of the structure. The structural form is changed imitating the behavior of basic multicellular units (BMU), thus the change concerns always the same amount of material (tissue), independently of the current values of SED according to formulas (18-20). Such a mechanism preserves the stability of the process and prevents rapid change in the form of the structure. The optimization process ceases when SED values on the whole surface of the structure are included in the "lazy zone". 
3.3. Numerical example. The developed optimization system based on trabecular bone remodeling was tested on a typical topology optimization example, the bending cantilever beam. The procedure, developed in opposition to standard optimization techniques, is capable of obtaining proper results (well known from literature [1]) without depending on the starting domain (Fig. 1).

The general time consuming issue of optimization process is finite element mesh generation for each step of structural evolution. The developed finite element mesh generator was originally dedicated to mesh creation for biological entities and is based on structure evolution. Since the visualization for the biological entities is based on the digital images e.g. Computed Tomography, the input to the system is also based on the collection of the 2 dimensional images. The images are obtained automatically by cutting a $3 \mathrm{D}$ CAD model, in a predefined direction, into a flat intersections. After preliminary graphical operations the images of slices are directly used for building of the 3-dimensional finite element mesh. The 2-dimensional image is first translated into the bitmap, where " 0 " represents void and " 1 " the object. On the bitmap the initial step of discretization is executed. The aim of this first step is to describe the areas with material. The discretization procedure produces a 2-dimensional network of tetragonal elements, according to the object image shape. The discretized 2-dimensional image is projected to the subsequent one. If there are areas containing material on both images, the boxes are created. Each box is in turn translated into 6 tetrahedral volume elements.

Thus, the remodeling process can be treated as a par excellence structural optimization method. Moreover, the method has unique features - domain independence, functional configurations during the process of optimization. The most important feature of the biomimetic optimization method is a fact that the multiple load case problem is implemented in the optimization scenario.

From an engineer's point of view the single load case problem is rather rare. More frequent, but also more valuable for mechanical design, is the problem of structural optimization under multiple loads. For the multiple load case simulation the same starting configuration (stick) was studied. Two different load cases were examined. The first, identical with the study presented in (Fig. 1), and the second, with the same definition of boundary conditions and horizontal bending force. The optimization results for these two configurations treated separately are depicted in (Fig. 2). The solutions have identical form, but rotated according to the direction of the force applied. (Fig. 3)

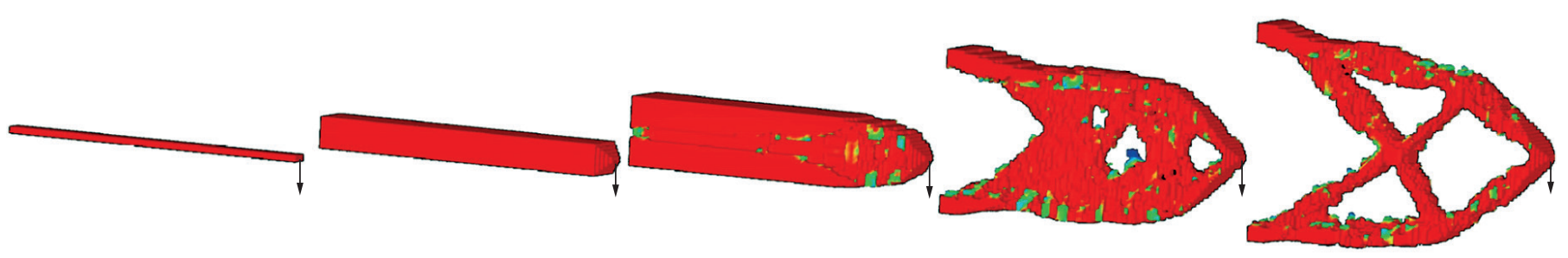

Fig. 1. Optimization results for a typical example (bending cantilever beam). Proper results obtained with the simplest connection between applied force and supports

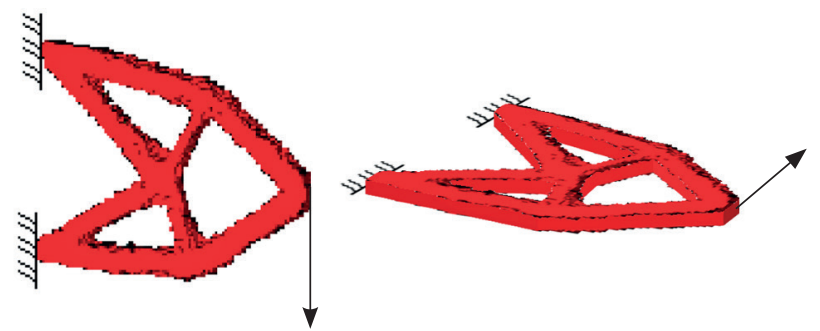

Fig. 2. The optimization results for the same starting configurations (stick) and different direction of the bending force: left - vertical bending, middle - horizontal bending force, right - superposition

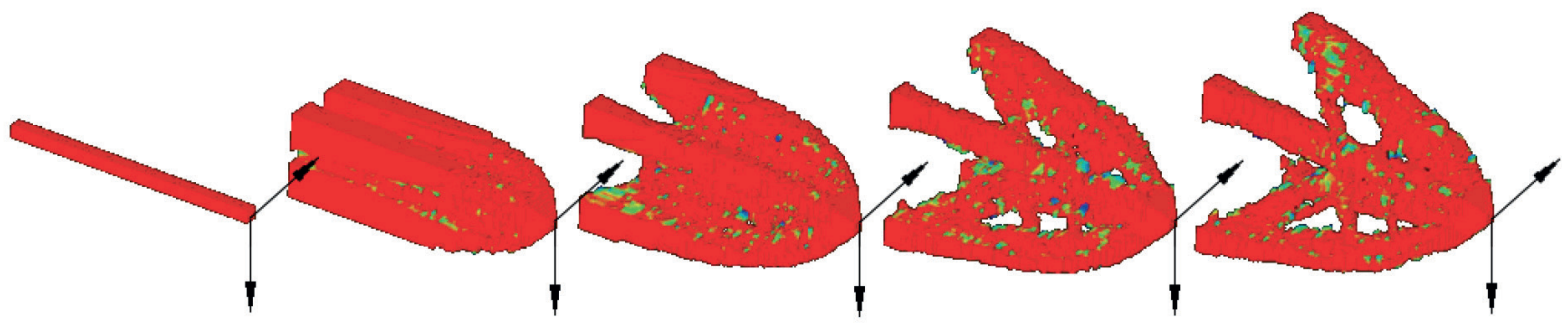

Fig. 3. The result of the multiple load study (altering vertical and horizontal bending forces) 
depicts the result for the same starting configuration but including multiple load cases. The direction of the force applied was switched every two simulation steps from the vertical to horizontal one and vice versa. The result of the multiple load study is presented in (Fig. 3). The solution obtained is radically different from those obtained for each of the load cases shown in (Fig. 2), nor is their superposition. Due to the unique features of biomimetic structural optimization process discussed above, the evolution of the structure proceeded smoothly, despite the changes in load definition. The method allows efficient performance of the optimization process for several cases of loading, when homogenization of SED on the surface of the structure guarantees the optimality of solution.

In the next chapters, a complex biomimetic optimization process of a symmetrical NACA0012 airfoil will be presented. The features special to the presented approach, allowing calculation of a multi-load case problem combining different flow conditions, are crucial for such task.

\section{Flow simulation}

In aerospace engineering, to obtain a proper balance between weight and strength of the internal wing structure, optimization techniques have to be used. On the other hand, in the process of designing an aircraft structure, the coupled fluid-structure interaction (FSI) simulations must be consid- ered as well. During the standard procedure, the topology optimization is performed separately from the size and shape optimization, due to differences in both methods. The method based on trabecular bone remodeling allows topology, size, and shape optimizations to be done altogether, simultaneously supplying them with modifiable loads from the aeroelastic analysis.

A system for coupling aeroelastic analysis [29] with structural optimization [30] has been developed. The presented approach is based on a loosely coupled technique, where each part of the multidisciplinary problem is solved independently and the results exchange between each other (Fig. 4).

At the beginning, the aerodynamic load based on the CFD simulation is computed. Afterwards the outer wing surface pressure distribution is interpolated on the structural mesh and the biomimetic structural optimization begins.

The pressure distribution interpolation between CFD and CSM calculations is imposed by the various discretization levels. Because of the non-matching grids, it is impossible to transfer the data directly from one structure to another. To ensure proper interpolation, a data exchange technique using coupling surface was implemented, enabling interaction between both grids. The calculated aerodynamic load is transferred from the CFD grid, through the coupling surface to the CSM mesh using the inverse distance weighting interpolation method. This approach guarantees that conservative values are maintained across computational procedures [31].

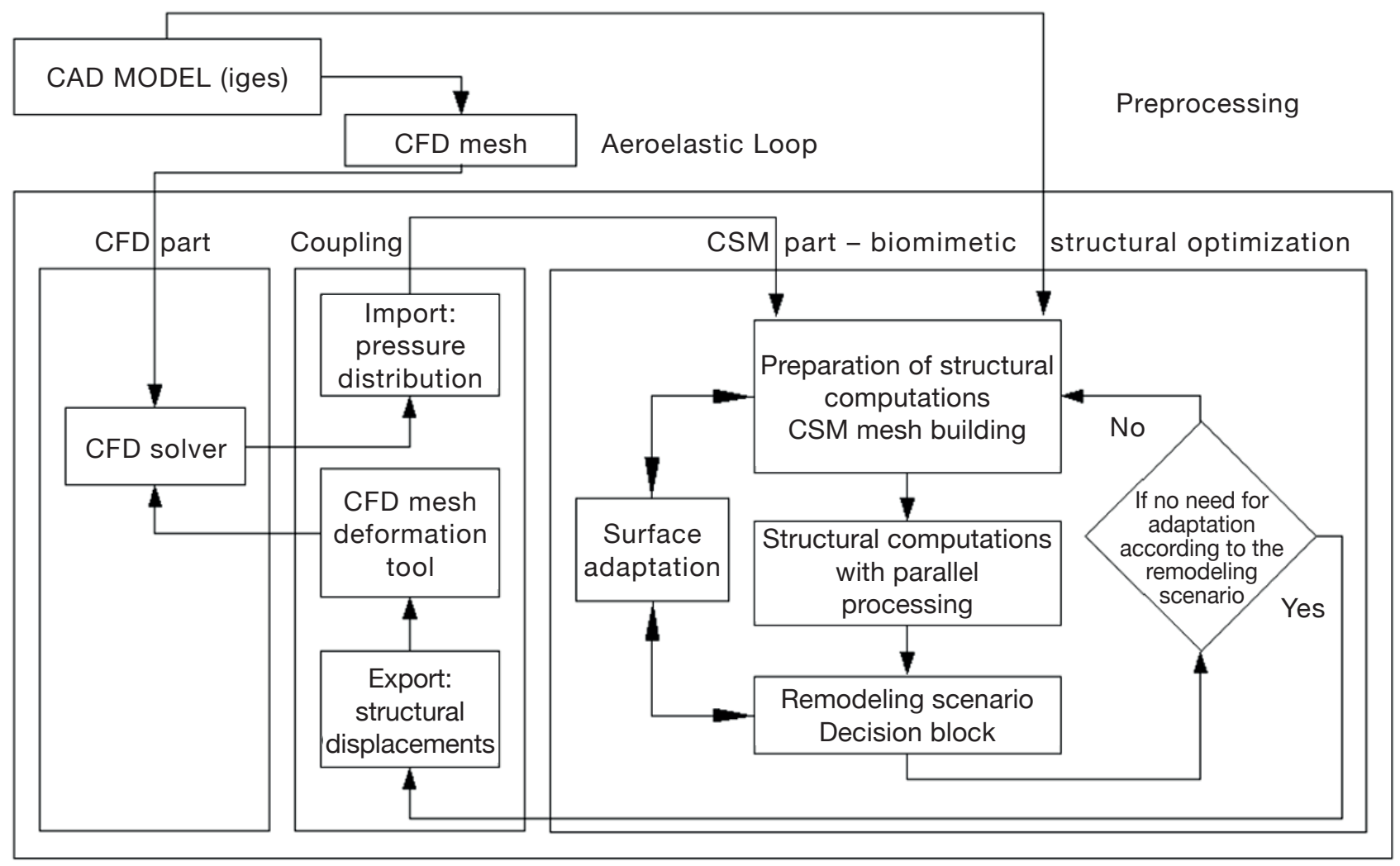

Fig. 4. The algorithm presenting coupling between aeroelastic analysis and biomimetic structural optimization 
(a)

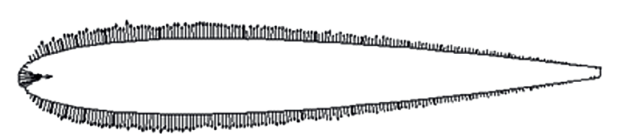

(b)

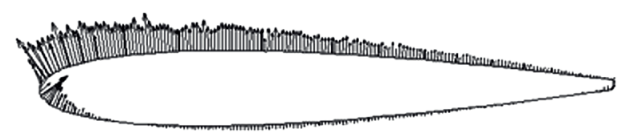

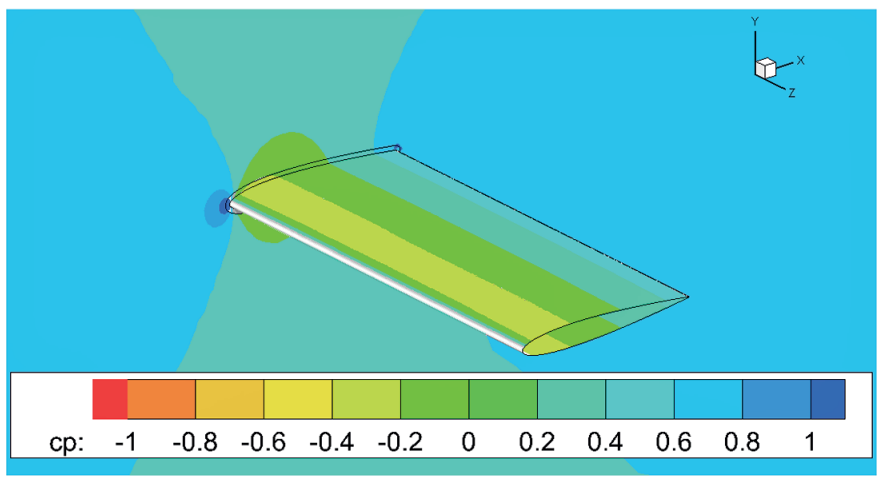

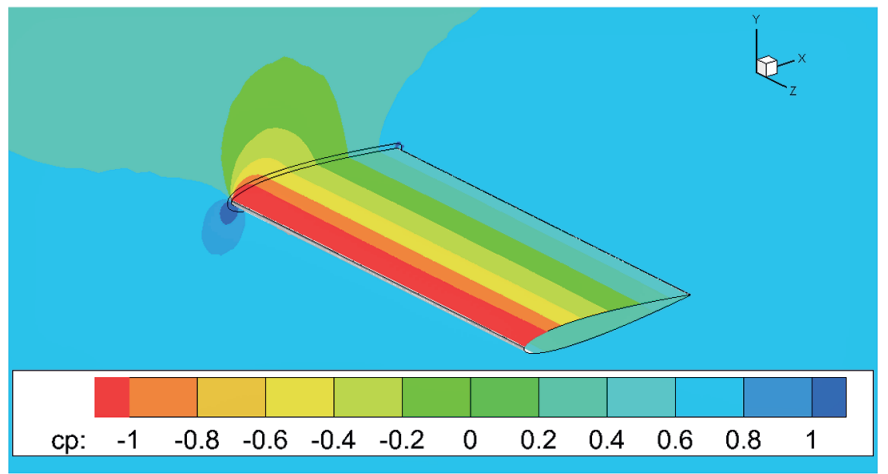

Fig. 5. Forces calculated in the aeroelastic environment (top) and coefficient pressure distribution (bottom) acting on the wing's surface under angles of attack $0^{\circ}$ (a) and $4^{\circ}$ (b) for the NACA0012 airfoil. The section of the wing is shown from the up. Cp is the difference between local static pressure and freestream static pressure, nondimensionalized by the freestream dynamic pressure

With an increasing angle of attack, aerodynamic forces, especially lifting force, also increase (Fig. 5). The location of the spars moves to the front of the airfoil and their volume increases to counter the growing torsional and bending moments from the lift and drag forces. Regardless of the increasing volume, the developed structure, even in the case of $4^{\circ}$ angle of attack, is much lighter than standard technological solutions.

\section{Numerical example}

In order to illustrate the biomimetic structural optimization method coupled with aeroelastic analysis, a multi load case problem of a straight wing with a symmetrical NACA0012 airfoil has been selected.

To increase the complexity of the solution, different flow conditions (including angle of attack reaching from 0 to 4 degrees and Mach number $\mathrm{Ma}=0.5$ ) were considered.

As it was presented in section 3 the unique feature of the method is also the possibility to include multiple load cases in a one optimization procedure. In the case of a trabecular bone remodeling the load applied to the structure is changing under external conditions however, it results in a single optimized bone structure. Those properties of biomimetic optimization were used for analysis with different angles of attack. The loads acting on a wing during the optimization process were alternately taken from the calculations made for $0^{\circ}$ and $4^{\circ}$ angles of attack. Thanks to the lazy zone, optimization procedure leads directly to defining a single final wing internal structure, being an optimal solution for both load cases.
To perform the CFD simulation of the wing's air flow and determine the pressure distribution on its surface, the C-shape CFD computational mesh, composed of almost 8 million elements, was generated. Stationary calculations were executed using Reynolds-averaged Navier-Stokes (RANS) equations, with the Spalart-Allmaras single-equation turbulence model in the standard version [29].

In order to transfer the aerodynamic loads from the CFD grid to the CSM mesh, a coupled surface model was created. The vectors representing pressure distribution were localized on 14 ribs distributed equally along the wing. The starting point of the biomimetic optimization was the empty inside wing configuration, with the thin material layer only, analogous to the NACA0012 airfoil. To obtain high quality results the wing's geometry was divided in a longitudinal direction into up to 400 two-dimensional sections with a distance of 0.001 meters between and element size of $0.0005 \times 0.0005$ meters. The largest FEM grid consisted of more than 4 million tetrahedral volume elements. For calculations, an isotropic material with Young's modulus $\mathrm{E}=6.9 \mathrm{GPa}$ and Poisson ratio $\mathrm{v}=0.33$ (corresponding to aluminum) was chosen. The lazy zone was specified in relation to material properties, including the Huber-Mises-Hencky stresses, minimal value of $10 \mathrm{MPa}$ and the maximal value of $100 \mathrm{MPa}$ was assumed.

\section{Results}

The selected steps of biomimetic optimization of the internal structure for the NACA0012 airfoil procedure for the angle of 


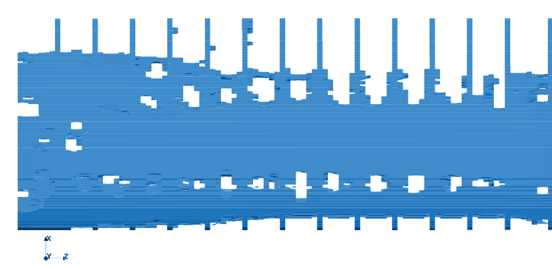

(a) Step: 8

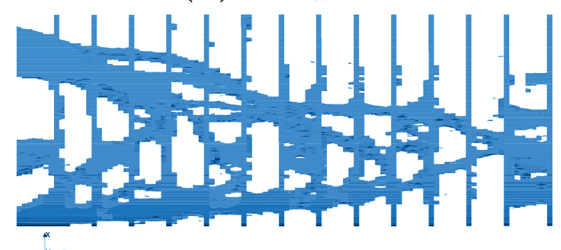

(d) Step: 70

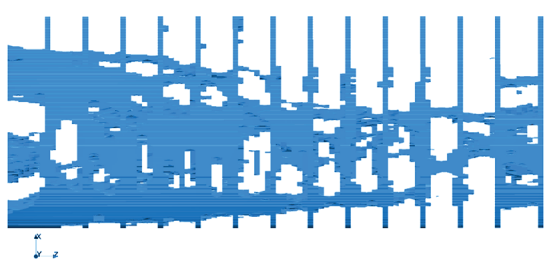

(b) 20

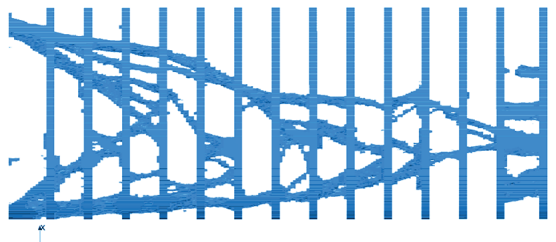

(e) 87

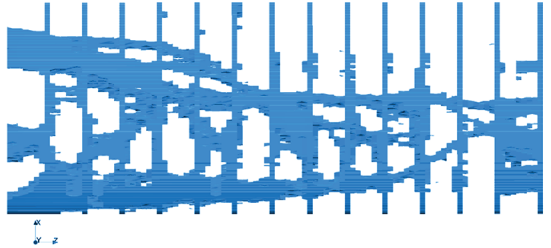

(c) 30

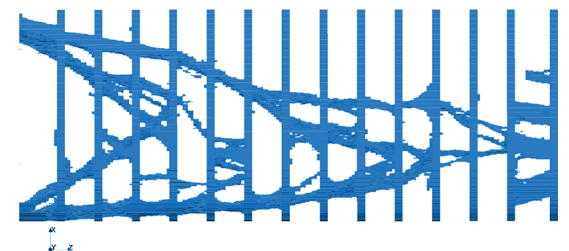

(f) 95

Fig. 6. Selected steps of the wing structural optimization process with the NACA0012 airfoil for angle of attack $0^{\circ}$ (Presented results can also be downloaded from: goo.gl/nBjJL3 and visualized as a 3DPDF in Free Adobe Reader)

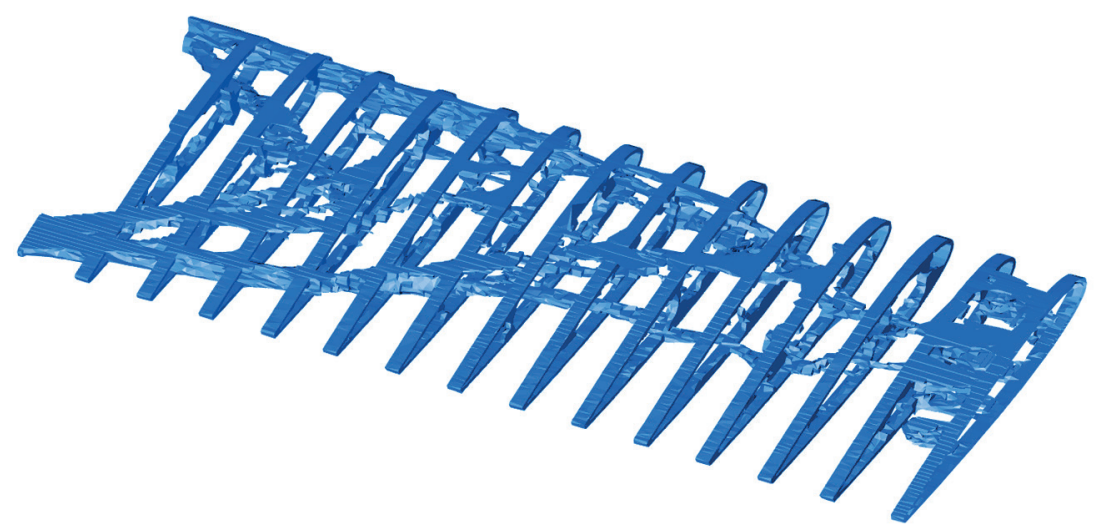

Fig. 7. 95th step of the wing structural optimization process with the NACA0012 airfoil for angle of attack $0^{\circ}$ (Presented results can also be downloaded from: goo.gl/nBjJL3 and visualized as a 3dPDF in Free Adobe Reader)

attack $0^{\circ}$ is presented (Fig. 6). The final step of the calculation when there is no need to change the structural form according to the assumptions is presented on (Fig. 7). It means that for all elements on the structural surface the strain energy density is between the lower and upper bounds of the lazy zone.
During the aeroelastic analysis, aerodynamic forces acting on the wing (lift and drag forces) were calculated (Table 1). The values for lift and drag forces increase in the angle of attack and change the aerodynamic moment to a positive one in relation to the aerodynamic center of the wing.

Table 1

Aerodynamic forces acting on the NACA0012 straight wing profile under different angles of attack

\begin{tabular}{|c|c|c|c|c|c|c|}
\hline \multicolumn{4}{|c|}{ Lift force $F_{l}[\mathrm{~N}]$} & \multicolumn{3}{|c|}{ Drag force $F_{d}[\mathrm{~N}]$} \\
\hline $\begin{array}{l}\mathrm{AOA} \\
\text { first }\end{array}$ & $\begin{array}{l}\text { Upper surface } \\
\text { second }\end{array}$ & $\begin{array}{c}\text { Lower surface } \\
\text { third }\end{array}$ & Total & Upper surface & Lower surface & Total \\
\hline $0^{\circ}$ & 273 & -273 & 0 & 15 & 13 & 28 \\
\hline $1^{\circ}$ & 345 & -201 & 144 & 9 & 19 & 28 \\
\hline $2^{\circ}$ & 418 & -133 & 285 & 4 & 25 & 29 \\
\hline $3^{\circ}$ & 495 & -68 & 427 & 0 & 32 & 32 \\
\hline $4^{\circ}$ & 574 & -7 & 567 & -2 & 39 & 37 \\
\hline
\end{tabular}


(a) $0^{\circ}$

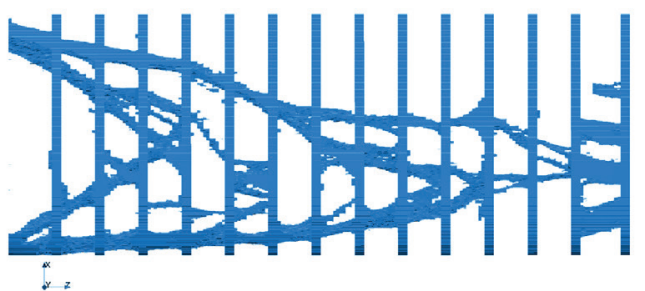

(b) $1^{\circ}$

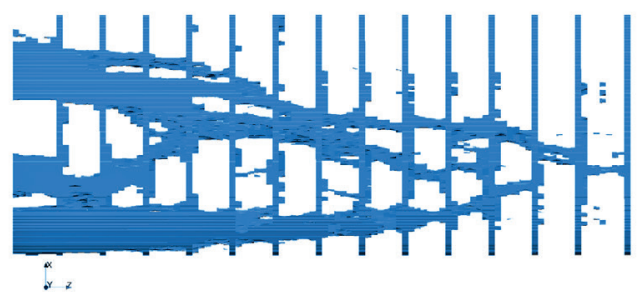

(c) $2^{\circ}$
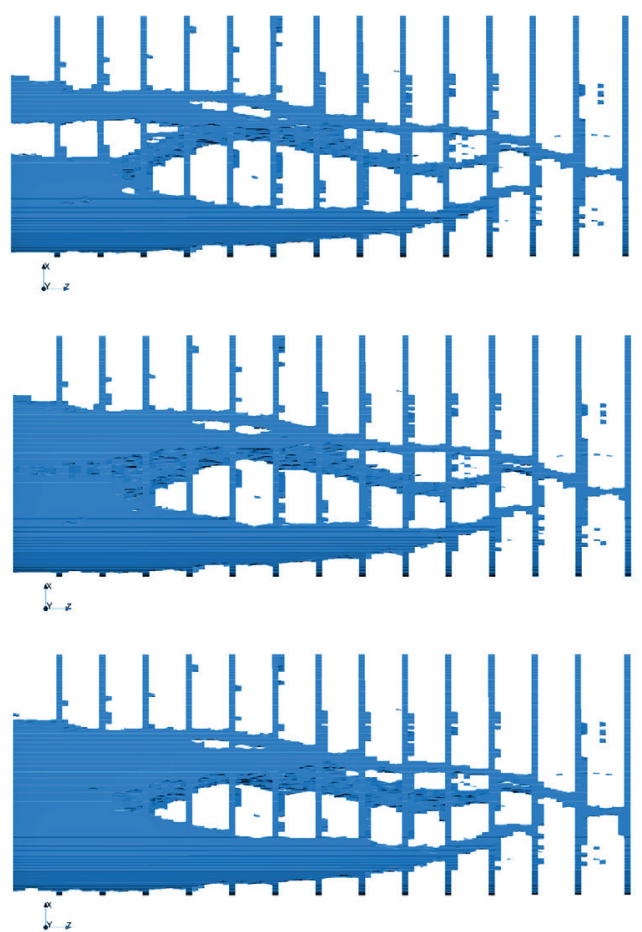

Fig. 8. Results of the wing biomimetic structural optimization with the NACA0012 airfoil under the angles of attack from $0^{\circ}$ to $4^{\circ}$
The distribution of the pressure ratio for angles of attack of $0^{\circ}$ and $4^{\circ}$ on the wing's surface is presented in (Fig. 5).

The study further included determining the distribution of material inside the wing for angles of attack reaching $0^{\circ}$ to $4^{\circ}$ (Fig. 8).

In the first analyzed case, due to the zero angle of attack, main vertical forces (lift forces) act on the wing in opposition, making the torsional and bending moments negligible. The structure created (Fig. 6f) prevents the skin from bursting by connecting the upper and lower parts of the ribs. The bending moment from drag force is compensated by longitudinal structures (spars) connecting the ribs (Fig. 7).

Additionally, an example of multi-load case problem combining the $0^{\circ}$ angle of attack case with the $4^{\circ}$ angle of attack case together has been calculated (Fig. 9). Due to the special capabilities of the biomimetic optimization method, it was possible to optimize the internal structure with different loads affecting the wing simultaneously [32]. The solution combines the advantages of the configurations of both cases, however, it is not overlapping one configuration over the other.

\section{Conclusions}

The multidisciplinary approach for combining structural and flow analysis with the biomimetic structural optimization presented here makes it possible to determine the optimal material distribution of the aircraft structures under specific flow conditions. The biomimetic optimization was based on the phenomenon of trabecular bone adaptation with two important features: the remodeling process occurred on the surface only, and mechanical stimulation was needed to maintain appropriate mass. The regulatory model was based on the concept of tissue adaptation and assumed the existence of homeostasis, described by the distinguished value of SED. In this case to obtain the optimal stiffest design, the energy density along the shape needed to be constant. To preserve balance, the lazy zone was introduced, preventing remodeling if the deviation of energy value was too small. Only significant changes in the mechanical stimulation resulted

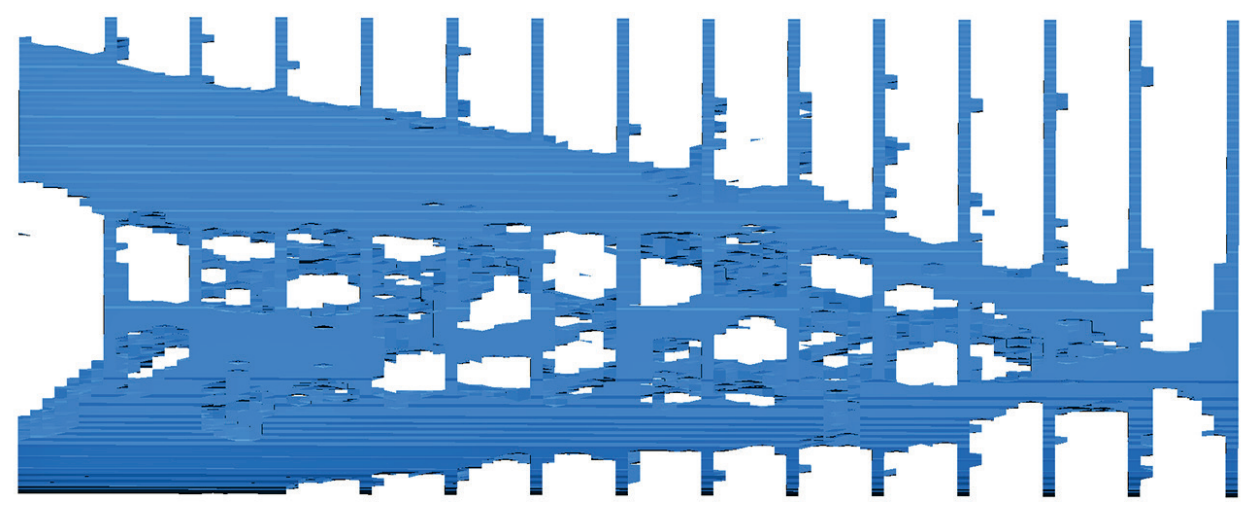

Fig. 9. Results of the wing biomimetic structural optimization with the NACA0012 airfoil under the combined parameters from angles of attack $0^{\circ}$ and $4^{\circ}-$ multi-load case solution 
in material loss or gain. This also stabilized the numerical procedure.

The selected test model - a wing with a symmetrical NACA0012 airfoil - was subjected to multiple aerodynamic loads at various angles of attack. Additionally, the configuration resulting from the multi-load case optimization, combining different flow conditions simultaneously, has been presented. The results obtained are similar to the solution presented by Sleesongsom and Bureerat [12], however this paper presents a discrete solution, using the ground structure. On the other hand, Oktay [11] presented optimum topology of the internal wing structure with assumed volume fraction. Further analysis needed for developing manufacturable structures was not made. To achieve such results, proper interpretation of the obtained structure is needed. The resulting volume presents an optimal structure with a constant energy density on its surface, guaranteeing the stiffest design.

Changing the angle of attack increases the lift force total values, generating bending and torsion moments. The biomimetic optimization method presented accommodates those moments by evolving the internal structure of the wing.

The presented method allows simultaneous optimization of size, shape, and topology of an aircraft structure under specific flow conditions, taking into account current interaction between the structure and the fluid. The structural volume resulting from the optimization process refers to assumed energetic lazy zone boundaries. The developed algorithm allows combination of multiple load cases in one computational case. Moreover, unlike most standard optimization techniques, the biomimetic approach doesn't need any domain to achieve proper results and allows functional configurations during the process of optimization. Additionally, this method solves the problem of a structure's scale by parallelization, allowing the mesh to be composed of up to $10^{6}$ elements. Such a solution offers a possibility of calculating more complex examples with high accuracy in reasonable time. The method presented sheds new light on of the potential for designing mechanical structures subjected under specified conditions in almost every branch of mechanical engineering.

To sum up, presented solution is a novelty in its field and has three main aspects: multi-physics aspect that involves two types of interaction (aero-elastic computational system for the simulation of interaction between the structural deflections and fluid flow, biomimetic structural optimization (the stiffest design principle) for structural form modification). Multi-processing aspect (both the aero-elastic system and the structural optimization environment is based on the parallel computing, including the mesh generation during the biomimetic optimization procedure). Biomimetic aspect in the structural optimization area (new possibilities of the structural optimization - simultaneous optimization of size, shape, and topology in the one numerical procedure, multiple load cases - something natural for the living entities - included in the new optimization procedure).

To obtain an efficient interpretation of these results, an accessible 3D visualization environment has been developed [33]. Presented results can also be downloaded from goo.gl/nBjJL3 and visualized as a 3DPDF in Free Adobe Reader.

\section{REFERENCES}

[1] S. Bendsoe, M. Philip, and O. Sigmund, Topology Optimization: Theory, Methods, and Applications, Springer Science \& Business Media, 2013.

[2] T. Lewiński, S. Czarnecki, G. Dzierżanowski, and T. Sokół, "Topology optimization in structural mechanics", Bull. Pol. Ac.: Tech. 61(1), 23-37 (2013).

[3] L. Wang, A. Williams, and R. Llamas, "Aircraft wing structural optimisation with manufacturing considerations", 8th Symposium on Multidisciplinary Analysis and Optimization (2000).

[4] L. Krog, A. Tucker, and G. Rollema, "Application of topology, sizing and shape optimization methods to optimal design of aircraft components", 3rd Altair UK HyperWorks Users Conference (2002).

[5] L. Krog, A. Tucker, M. Kemp, and R. Boyd, "Topology optimization of aircraft wing box ribs", 10th AIAA/ISSMO Multidisciplinary Analysis and Optimization Conference (2004).

[6] M. Stettner and G. Schuhmacher, "Optimization assisted design of military transport aircraft structures", Altair Optimization Technology Conference OTC04, Troy (2004).

[7] K. Maute and M. Allen, "Conceptual design of aeroelastic structures by topology optimization", Structural and Multidisciplinary Optimization 27(1), 27-42 (2004).

[8] S. Liu, X. An, and H. Jia, "Topology optimization of beam cross-section considering warping deformation", Structural and Multidisciplinary Optimization 35(5), 403-411 (2008).

[9] K. A. James, J. Martins, and J. Hansen, "Threedimensional structural topology optimization of an aircraft wing using level set methods", 12th AIAA/ISSMO Multidisciplinary and Optimization Conference, Victoria (2008).

[10] E. Oktay, H. Akay, and O. Merttopcuoglu, "Parallelized structural topology optimization and CFD coupling for design of aircraft wing structures", Computers \& Fluids 49(1), 141-145 (2011).

[11] E. Oktay, H.U. Akay, and O.T. Sehitoglu, "Threedimensional structural topology optimization of aerial vehicles under aerodynamic loads", Computers \& Fluids 92, 225-232 (2014).

[12] S. Sleesongsom and S. Bureerat, "New conceptual design of aeroelastic wing structures by multi-objective optimization", Engineering Optimization 45(1), 107-122 (2013).

[13] R. Huiskes, R. Ruimerman, G.H. Van Lenthe, and J.D. Janssen, "Effects of mechanical forces on maintenance and adaptation of form in trabecular bone", Nature 405(6787), 704-706 (2000).

[14] G.L. Niebur, M.J. Feldstein, J.C. Yuen, T.J. Chen, and T.M. Keaveny, "High-resolution finite element models with tissue strength asymmetry accurately predict failure of trabecular bone", Journal of Biomechanics 33(12), 1575-1583 (2000).

[15] K. Tsubota, T. Adachi, and Y. Tomita, "Functional adaptation of cancellous bone in human proximal femur predicted by trabecular surface remodeling simulation toward uniform stress state", Journal of Biomechanics 35(12), 1541-1551 (2002).

[16] R. Ruimerman, B. van Rietbergen, P. Hilbers, and R. Huiskes, "A 3-dimensional computer model to simulate trabecular bone metabolism", Biorheology 40(1, 2, 3), 315-320 (2003).

[17] M. Nowak, "From the idea of bone remodelling simulation to parallel structural optimization", Numerical Methods for Differential Equations, Optimization, and Technological Problems, Springer, 335-344 (2013).

[18] M. Nowak, "A generic 3-dimensional system to mimic trabecular bone surface adaptation", Computer Methods in Biomechanics and Biomedical Engineering 9(5), 313-317 (2006).

[19] W. Roux, Gesammelte Abhandlungen über Entwicklungsmechanik der Organismen II, Leipzig, 1895. 
D. Gawel, M. Nowak, H.'Hausa, and R. Roszak

[20] F. Pauwels, Biomechanics of the Locomotor Apparatus, SpringerVerlag, Berlin, 1963.

[21] D.R. Carter, T.E. Orr, and D.P. Fyhrie, "Relationships between loading history and femoral cancellous bone architecture", Journal of Biomechanics 22(3), 231-244 (1989).

[22] H.M. Frost, Laws of Bone Structure, Springfield, Ill: Charles C. Thomas, 1964.

[23] J. Telega, A. Galka, and S. Tokarzewski, "Effective moduli of trabecular bone", Acta Bioeng Biomech 1(1), (1999).

[24] R. Huiskes, Computational Theories of Bone Modeling and Remodeling, Advanced Course on Modelling in Biomechanics, Institute of Fundamental Technological Research, Polish Academy of Sciences, Warsaw, 2003.

[25] R. Huiskes, H. Weinans, J. Grootenboer, M. Dalstra, M. Fudala, and T. J. Slooff, "Adaptive bone remodelling theory applied to prosthetic- design analysis", Journal of Biomechanics 20, 11351150 (1987).

[26] Z. Wasiutynski, "On the congruency of the forming according to the minimum potential energy with that according to equal strength”, Bull. Pol. Ac.: Tech 8(6), 259-268 (1960).
[27] K. Dems and Z. Mroz, "Multiparameter structural shape optimization by finite element method", Int. J. Num. Meth. Eng. 13, 247-263 (1978).

[28] P. Pedersen, "Optimal designs-structures and materials problems and tools", 2003.

[29] R. Roszak, P. Posadzy, W. Stankiewicz, and M. Morzynski, "Fluidstructure interaction for large scale complex geometry and non-linear properties of structure", Archives of Mechanics 61(1), 3-27 (2009).

[30] M. Nowak, "Structural optimization system based on trabecular bone surface adaptation", Structural and Multidisciplinary Optimization 32(3), 241-249 (2006).

[31] H. Hausa, M. Nowak, and R. Roszak, "The coupled aeroelastic and structural optimization environment", 21st Fluid Mechanics Conference, Krakow (2014).

[32] M. Nowak, "On some properties of bone functional adaptation phenomenon useful in mechanical design", Acta Bioeng Biomech 12(2), 49-54 (2010).

[33] D. Gaweł and M. Nowak, "Accessible visualisation of topology optimisation results", 20th International Conference on Computer Methods in Mechanics, Poznan (2013). 